\title{
Les indicateurs d'inclusion sociale : où est la Wallonie aujourd'hui ?
}

\author{
Cécile Atta, Claire Maréchal et Sergio Perelman* \\ CREPP, Université de Liège
}

\begin{abstract}
We use the primary indicators of social inclusion proposed within the scope of the Open Method of Coordination (OMC) to situate Wallonia in the European Union (EU15) context. The results show that Wallonia stood in 2005 at the same level as Greece and was behind the EU15 level mainly regarding poverty and long-term unemployment rates. Groups at risk are single-parent families as well as unemployed and other inactive categories. Having at disposal these indicators for the years to come will be a must not only for the design but also for the evaluation of policies towards a better social cohesion.
\end{abstract}

Key words - social inclusion, Wallonia, poverty, housing.

\begin{abstract}
Résumé - Dans cet article, nous utilisons les indicateurs primaires d'inclusion sociale proposés dans le cadre de la Méthode de Coordination Ouverte (MOC), pour situer la Wallonie dans le contexte de l'Europe des 15 (UE15). Les résultats montrent qu'elle se situait en 2005 au même niveau que la Grèce, et qu'elle présentait un certain retard principalement au niveau du taux de pauvreté et du chômage de long terme. Les groupes à risque sont les familles monoparentales, les chômeurs ainsi que d'autres catégories d'inactifs. La possibilité de disposer de ces indicateurs de manière systématique est un atout important pour l'orientation des politiques en faveur d'une plus grande cohésion sociale, mais également pour l'évaluation de ces mêmes politiques.
\end{abstract}

Mots clés - inclusion sociale, Wallonie, pauvreté, logement.

\footnotetext{
* Les auteurs tiennent à remercier le soutien financier de la Communauté Française de Belgique (Action de Recherche Collective, ARC 05/10-332) ainsi que le SPF Sécurité Sociale pour avoir mis à notre disposition les informations statistiques recueillies par le Groupe de travail Indicateurs (PANincl).
} 


\section{INTRODUCTION}

L'Union européenne s'est dotée en l'année 2000, lors du Conseil de Lisbonne, de la Méthode de Coordination Ouverte (MOC) en matière d'exclusion sociale. À cette occasion, l'ensemble des pays ont choisi de manière concertée une série d'indicateurs qui doivent être mesurés de façon identique dans tous les pays et publiés régulièrement. Ces indicateurs, connus également sous le nom d'Indicateurs de Laeken ${ }^{1}$, sont censés devenir au fil du temps la base d'une méthode de comparaison volontaire, avec l'espoir que " par comparaison et par émulation, chaque pays se rapproche des meilleures pratiques ${ }^{2}$.

Le développement de la MOC a ainsi permis de rassembler une batterie unique d'indicateurs d'exclusion couvrant plusieurs domaines : revenu, emploi, éducation, logement et santé. La plupart de ces indicateurs sont calculés sur base d'enquêtes auprès de la population. Malheureusement, la taille de l'échantillon bruxellois est insuffisante pour obtenir des indicateurs fiables pour cette région.

Dans cet article, nous utiliserons un sous-ensemble de ces indicateurs afin de situer la Région Wallonne, et la Flandre, dans le contexte de la MOC. II s'agit d'un exercice hypothétique, les entités régionales n'ayant pas adhéré en tant que telles à la MOC, mais qui pourrait servir, on peut l'espérer, à la fixation d'objectifs régionaux en matière d'inclusion sociale ${ }^{3}$.

Cette note est divisée en trois sections. Dans la première section, nous nous intéressons aux cinq indicateurs primaires d'inclusion sociale inscrits dans la MOC et nous regardons où se situe la Région wallonne par rapport à la Flandre et aux pays qui composent l'Europe des 15 (UE15). La deuxième section est consacrée à une analyse détaillée de l'indice de pauvreté selon différents critères - sexe, âge, statut d'activité et type de ménage - de façon à identifier des groupes à risque au sein de la population wallonne, en particulier ceux dont la situation dépend essentiellement des transferts sociaux. Dans la troisième section, nous abordons la thématique du logement qui constitue un facteur déterminant d'inclusion sociale. Enfin, à titre de conclusion, nous terminerons par une série de réflexions et de suggestions pouvant servir à alimenter le débat d'idées sur l'orientation des politiques de lutte contre l'exclusion sociale.

\section{LES INDICATEURS DE LAEKEN}

Sur le tableau 1 sont présentées les valeurs correspondant à cinq indicateurs, choisies parmi les indicateurs primaires d'inclusion sociale : taux de pauvreté, inégalité de revenus, chômage de longue durée, désertion scolaire et espérance de vie. Les définitions exactes de ces indicateurs sont présentées en annexe. Les chiffres présentés correspondent à ceux calculés pour l'année 2005.

1. Voir Atkinson et al. (2001) pour une justification de la sélection de ces indicateurs et leur définition.

2. Lefebvre et al. (2005).

3. Dans certains écrits et documents, on trouve le terme " exclusion sociale " et dans d'autres celui d'« inclusion sociale " pour se référer au même phénomène. Nous les utiliserons dès lors ici comme synonymes, étant sous entendu qu'ils font allusion aux deux faces d'une même pièce. 
De manière générale, sur l'ensemble des domaines couverts par ces indicateurs la situation de la Région wallonne est défavorable par rapport à celle de la Région flamande. Elle l'est également par rapport à la Belgique, à l'exception de l'indicateur d'inégalité des revenus qui est plus favorable au niveau des régions qu'au niveau national. Ce résultat s'explique en partie par les différences existantes entre les revenus moyens des régions, mais également par une inégalité plus grande de revenus au sein de la Région bruxelloise ${ }^{4}$.

Par rapport aux valeurs moyennes de I'UE15, la Wallonie affiche une situation favorable en termes d'inégalité de revenus et de désertion scolaire mais défavorable en termes de taux de pauvreté, d'espérance de vie et du chômage de longue durée, tout particulièrement dans ce dernier cas où elle obtient un score plus de deux fois supérieur à la moyenne, 6,9 \% et 2,7\%, respectivement.

Peut-on avoir une vision d'ensemble de l'exclusion sociale ?

S'agissant d'une problématique multidimensionnelle, la réponse n'est pas immédiate, et surtout pas unique. En effet, plusieurs indicateurs synthétiques sont proposés dans la littérature, soit en pondérant au préalable chacun des indicateurs primaires, soit par des méthodes qui font appel à des pondérations implicites, comme c'est le cas des méthodes des frontières d'efficacité. L'objet de cette note n'étant pas d'entrer dans ce débat, nous adopterons ici une méthodologie simple qui consiste à calculer un indicateur synthétique moyen non pondéré (Lefebvre et al., 2005).

Cet indicateur est présenté dans la dernière colonne du tableau 1. II est obtenu en normalisant chacun des indicateurs sur l'intervalle 0-1 avec comme extrêmes les valeurs minima et maxima (l'indicateur d'espérance de vie est préalablement inversé) et en faisant la moyenne de ces valeurs ensuite. Procédant de cette manière, l'indicateur synthétique obtenu a comme caractéristique de donner une pondération identique à l'intervalle de variation observé pour chacun des indicateurs primaires : pauvreté, inégalité, chômage, désertion scolaire et espérance de vie.

Ce qu'on observe tout d'abord, c'est que l'indicateur synthétique moyen pour la Belgique $(0,58)$ se situe légèrement sous la moyenne de l'UE15 $(0,64)$, mais que les régions s'en éloignent dramatiquement. D'une part, la Flandre atteint un des meilleurs scores $(0,86)$, juste après la Suède qui présente l'indicateur le plus élevé $(0,95)$. D'autre part, la Wallonie obtient un indicateur synthétique bien plus bas que la moyenne $(0,39)$ et comparable à celui de la Grèce $(0,37)$.

Que peut-on conclure de ces observations?

II y a certainement au moins deux facteurs qui jouent un rôle déterminant dans l'exclusion sociale en Wallonie. II s'agit du risque de pauvreté et de la difficulté à sortir des situations de non emploi. C'est un premier diagnostic. II mérite que l'on s'attarde à l'analyser plus en détail, en particulier que l'on regarde quels sont les groupes à risque de pauvreté. C'est l'objet de la section suivante où nous essayons d'identifier les catégories les plus exposées dans la population.

4. Malheureusement on dispose de peu d'informations sur la situation en région bruxelloise. On peut néanmoins citer le cas de l'indicateur de pauvreté, 29,6 \% en 2005, qui place Bruxelles très loin derrière la Flandre et la Wallonie sur ce plan. 
Tableau 1 : Indicateurs d'inclusion sociale en 2005

\begin{tabular}{|c|c|c|c|c|c|c|}
\hline $\begin{array}{c}\text { Pays } \\
\text { Régions }\end{array}$ & $\begin{array}{c}\text { Taux de } \\
\text { pauvreté } \\
(\%)\end{array}$ & $\begin{array}{l}\text { Inégalité } \\
\text { de revenus } \\
\text { (S80/S20) }\end{array}$ & $\begin{array}{l}\text { Chômage } \\
\text { de longue } \\
\text { durée (\%) }\end{array}$ & $\begin{array}{l}\text { Désertion } \\
\text { scolaire (\%) }\end{array}$ & $\begin{array}{l}\text { Espérance } \\
\text { de vie } \\
\text { (années) }\end{array}$ & $\begin{array}{l}\text { Indicateur } \\
\text { synthétique } \\
\text { moyen }\end{array}$ \\
\hline Allemagne & 12 & 3,8 & 5,7 & 13,8 & 79,4 & 0,61 \\
\hline Autriche & 12 & 3,8 & 1,3 & 9.0 & 79,6 & 0,81 \\
\hline Belgique & 15 & 4,0 & 4,4 & 13.0 & 79,1 & 0,58 \\
\hline Flandre & 11 & 3,5 & 2,2 & 10,7 & 80,4 & 0,86 \\
\hline Wallonie & 17 & 3,7 & 6,9 & 14,6 & 78,1 & 0,39 \\
\hline Danemark & 12 & 3,5 & 1,1 & 8,5 & 78,3 & 0,74 \\
\hline Espagne & 20 & 5,4 & 2,2 & 30,8 & 80,3 & 0,45 \\
\hline Finlande & 12 & 3,6 & 2,2 & 9,3 & 79,1 & 0,75 \\
\hline France & 13 & 4,0 & 3,8 & 12.0 & 80,3 & 0,72 \\
\hline Grèce & 20 & 5,8 & 5,1 & 13,3 & 79,2 & 0,37 \\
\hline Irlande & 20 & 5 & 1,5 & 12,3 & 79,5 & 0,56 \\
\hline Italie & 19 & 5,6 & 3,9 & 21,9 & 81.0 & 0,50 \\
\hline Luxembourg & 13 & 3,8 & 1,2 & 13,3 & 79,6 & 0,76 \\
\hline Pays-Bas & 11 & 4.0 & 1,9 & 13,6 & 79,6 & 0,76 \\
\hline Portugal & 19 & 6,9 & 3,7 & 38,6 & 78,1 & 0,13 \\
\hline Royaume-Uni & 19 & 5,8 & 1.0 & 14.0 & 79,1 & 0,51 \\
\hline Suède & 9 & 3,3 & 1,2 & 11,7 & 80,7 & 0,95 \\
\hline UE15 & 15 & 4,6 & 2,7 & 15,7 & 79,5 & 0,64 \\
\hline
\end{tabular}

Source : Eurostat, Indicateurs communs ; Calculs propres.

\section{LES CATÉGORIES À RISQUE DE PAUVRETÉ}

Lorsque l'on parle de pauvreté en Europe, on fait en général référence à une notion relative. C'est cet aspect qui a été retenu dans les indicateurs de Laeken. II est justifié en deux temps par Atkinson et al. (2001). D'une part, dans nos pays développés, il s'agit plutôt de partager entre les individus les bénéfices d'une certaine croissance économique que d'assurer un minium vital, comme c'est le cas pour les pays en développement. D'autre part, la pauvreté dépend fortement du cadre de vie et du développement socio-économique dans lequel l'individu évolue. Élaborer une mesure de la pauvreté qui serait pertinente dans tous les endroits du monde serait insensé.

C'est la raison pour laquelle le taux de pauvreté choisi comme indicateur de base par la MOC utilise comme seuil de pauvreté les $60 \%$ du revenu médian national. Le revenu est défini comme le revenu disponible du ménage ajusté au moyen d'une échelle d'équivalence qui tient compte des économies d'échelle potentielles au niveau des besoins de base. On utilise une échelle d'équivalence construite de la manière suivante : 1,0 pour le chef du ménage, $+0,5$ pour les autres personnes faisant partie du ménage et âgées de 15 ans et plus, et + 0,3 pour chaque enfant âgé de moins de 15 ans. 
Le tableau 2 présente les taux de pauvreté observés en 2005 pour différentes catégories de la population au sein des Régions wallonne et flamande, ainsi que pour la Belgique et pour l'Europe des 15. Les pourcentages sont obtenus en comptant le nombre d'individus qui se trouvent sous le seuil de pauvreté dans chaque catégorie. Par construction, toutes les personnes appartenant à un ménage ayant été identifié comme pauvre sont également considérées pauvres. Les moyennes européennes correspondent aux moyennes non pondérées des taux de pauvreté au niveau national.

\section{Qu'observe-t-on sur ce tableau?}

Tout d'abord, comme on pouvait s'y attendre, les taux de pauvreté sont systématiquement supérieurs en Wallonie qu'en Flandre, à une exception près, celle des personnes âgées de 65 ans et plus vivant en couple. Ensuite, on remarque qu'une proportion plus grande de femmes que d'hommes se trouve dans une situation de pauvreté. Ceci vaut pour la Belgique mais également pour l'UE15, l'écart étant cependant relativement plus important en Wallonie qu'ailleurs.

La structure de la pauvreté par âge montre une situation particulièrement contrastée entre la Belgique et l'UE15. En Belgique, on remarque une diminution systématique des taux de pauvreté avec l'âge, par contre au niveau de l'Europe c'est la catégorie des aînées (65 ans et plus) qui apparaît comme la plus exposée à ce risque. Mais la situation est aussi contrastée entre la Flandre et la Wallonie. En Flandre, on observe une diminution de moitié entre les deux catégories d'âge extrêmes (12,4 \% pour les 0-17 et 6,7 pour les 65+), par contre en Wallonie la pauvreté parmi les plus âgés reste à un niveau relativement élevé (15,3\%) comparée à la catégorie des moins de 18 ans (20,2 \%).

Ces constatations sont conformes aux résultats obtenus pour la Wallonie par Guio $(2003,2006)$ pour des années récentes. Par contre, elles diffèrent de ceux rapportés dans Delhausse et al. $(1999,2002)$ qui faisaient apparaître une situation beaucoup plus favorable, en termes relatifs, des personnes âgées au milieu des années 1990. Ceci est sans doute l'illustration d'une paupérisation rapide de cette tranche de la population wallonne au cours de la dernière décennie.

On ne manquera pas d'observer aussi un autre phénomène préoccupant, les taux de pauvreté relativement élevés pour des catégories d'âge intermédiaires, notamment celle des 25-49 ans, c'est-à-dire en plein âge actif. Sachant les difficultés existants au niveau de l'intégration sur le marché de l'emploi en Wallonie, déjà soulignées auparavant par l'observation d'un taux de chômage de longue durée particulièrement élevé, nous nous sommes intéressés aux taux de pauvreté selon le statut d'activité.

Sans surprise, c'est parmi les personnes qui occupent un emploi que I'on trouve les taux de pauvreté les plus faibles. Le taux de pauvreté des actifs en Belgique (3,9 \%) est d'ailleurs inférieur à celui de l'UE15 (7,0 \%). Même si l'on doit constater à partir de ces résultats l'émergence d'une catégorie de travailleurs pauvres en Belgique, elle reste relativement faible comparativement à d'autres pays (Guio, 2006). Cependant, on observe au sein de la catégorie des travailleurs indépendants un risque plus élevé de pauvreté, plus important en Wallonie (14,9\%) qu'en Flandre (9,9\%). 
C'est finalement parmi les catégories d'inactifs, définies au sens large, que devrait par déduction se concentrer la population en situation de pauvreté. C'est ce que l'on constate dans le tableau 2 mais avec des différences selon les situations. On observe en effet que le pourcentage de retraités vivant dans des ménages situés en dessous du seuil de pauvreté ne diffère pas substantiellement entre la Belgique et l'Europe des 15, ni au niveau des Régions wallonne et flamande. Par contre, c'est dans les catégories des personnes en situation de chômage ou inactives (hors retraite), que les taux de pauvreté atteignent des pourcentages très élevés, particulièrement en Wallonie, mais également au niveau de l'UE15. Entre la Flandre et la Wallonie, les chiffres varient dramatiquement avec des taux de 36,0 \% et 28,8 \% pour les chômeurs et autres inactifs en Wallonie, contre 19,1 \% et $18,0 \%$ respectivement en Flandre.

Tableau 2 : Taux de pauvreté en 2005 par sexe, âge, profession et type de ménage

\begin{tabular}{|c|c|c|c|c|c|}
\hline Catégorie & Classes & Wallonie & Flandre & Belgique & UE15 \\
\hline \multirow[t]{2}{*}{ Sexe } & Hommes & 15.7 & 10.7 & 14.1 & 14,3 \\
\hline & Femmes & 17.9 & 11.9 & 15.5 & 15,8 \\
\hline \multirow[t]{5}{*}{ Âge } & $0-17$ & 20,2 & 12,4 & 18,1 & 17 \\
\hline & $18-24$ & 19,3 & 12,0 & 17,0 & 19 \\
\hline & $25-49$ & 17,8 & 11,3 & 16,3 & 12 \\
\hline & $50-64$ & 14,8 & 8,4 & 12,3 & 13 \\
\hline & $65+$ & 15,3 & 6,7 & 11,4 & 19 \\
\hline \multirow{6}{*}{$\begin{array}{l}\text { Statut } \\
\text { d'activité }\end{array}$} & Actifs & 3,9 & 3,2 & 3,9 & 7 \\
\hline & - travailleur salarié & 2,3 & 2,3 & 2,8 & - \\
\hline & - indépendant & 14,9 & 9,9 & 12,4 & - \\
\hline & Chômeurs & 36,0 & 19,1 & 30,7 & 37 \\
\hline & Retraités & 18,9 & 19,4 & 19,1 & 17 \\
\hline & Autres inactifs & 28,8 & 18,0 & 24,4 & 26 \\
\hline \multirow{11}{*}{$\begin{array}{l}\text { Type de } \\
\text { ménage }\end{array}$} & Sans enfants dépendants & 15,4 & 11,9 & 13,8 & 15 \\
\hline & - 1 personne & 23,9 & 19,3 & 22 & 26 \\
\hline & -2 personnes $<65$ ans & 10,0 & 6,3 & 8,4 & 9 \\
\hline & - 2 personnes, au moins une $65+$ ans & 14,9 & 19,3 & 17,4 & 15 \\
\hline & - autres & 8,8 & 2,7 & 4,5 & 8 \\
\hline & Avec enfants dépendants & 17,9 & 10,8 & 15,7 & 15 \\
\hline & - ménage monoparental & 39,4 & 19,3 & 32,1 & 32 \\
\hline & - couple +1 enfant & 14,0 & 5,7 & 9,3 & 10 \\
\hline & - couple +2 enfants & 15,2 & 5,9 & 9,8 & 12 \\
\hline & - couple +3 enfants ou + & 11,9 & 18,6 & 18,9 & 22 \\
\hline & - autres & 18,5 & 11,9 & 19,0 & 15 \\
\hline Total & & 16,8 & 11,3 & 14,8 & 15,1 \\
\hline
\end{tabular}

Source : Groupe de travail Indicateurs PANincl, SPF Sécurité Sociale. À partir des données recueillies dans le cadre de EU-SILC (European Union - Survey on Income and Living Conditions). 
Comment expliquer ces différences sachant que les règles qui fixent l'octroi et le calcul du montant des allocations sociales sont identiques dans les deux régions?

En règle générale, les montants des revenus de remplacement, pensions et allocations de chômage, maladie ou invalidité, ont été conçus pour couvrir les besoins d'une personne isolée, ou d'un ménage le cas échéant. Néanmoins, comme en témoigne la figure 1, dans un certain nombre de pays ces montants sont inférieurs aux seuils de pauvreté. C'est le cas de la Belgique où ils se situent autour de $70 \%$ du seuil de pauvreté.

Figure 1 : Adéquation des allocations sociales avec le seuil de pauvreté en 2006 (UE-15)

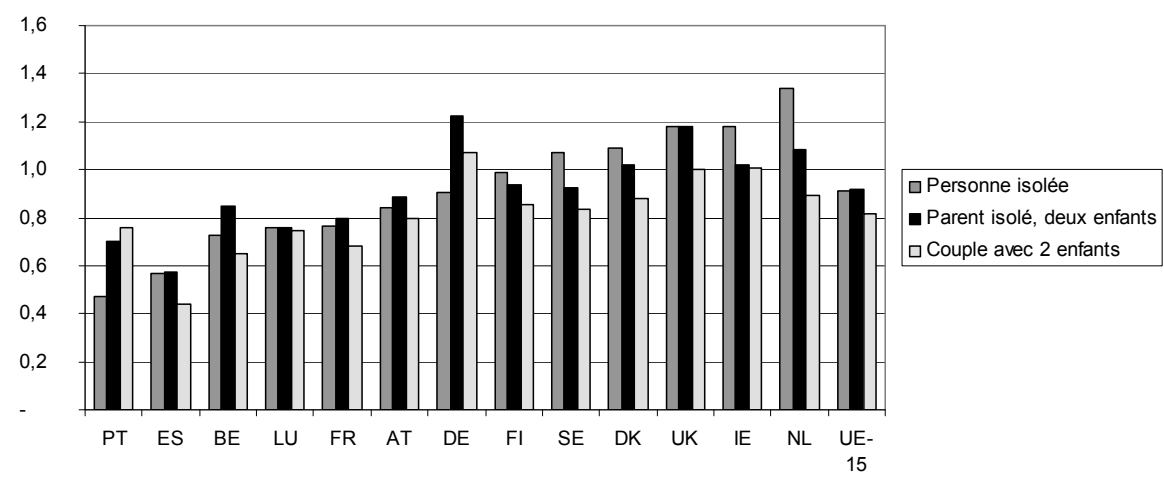

Source : Groupe de travail Indicateurs PANincl., SPF Sécurité Sociale.

Ceci dit, qu'est-ce qui fait la différence entre les allocataires sociaux wallons et flamands face au risque de pauvreté ?

La réponse à cette question se trouve certainement dans la manière dont une situation individuelle, celle des allocataires de revenus de remplacement, se combine avec celle des autres personnes du ménage. Ainsi, une personne bénéficiaire de revenus de remplacement qui cohabite avec une ou plusieurs personnes ayant des revenus professionnels échappera dans la plupart des cas au risque de pauvreté.

Ceci nous amène naturellement à nous intéresser au risque de pauvreté par type de ménage. En bas du tableau 2 sont présentés les taux correspondant aux ménages avec et sans enfants dépendants. On n'observe pas des différences significatives entre ces deux catégories de ménages, par contre on constate des différences importantes dans leur sein.

Ainsi, parmi les ménages sans enfants, ce sont les personnes seules qui sont le plus touchées par le risque de pauvreté. On remarque aussi que les couples âgés (dont une personne au moins a plus de 65 ans) sont plus exposés à la pauvreté en Flandre (19,3\%) qu'en Wallonie (14,9\%). Ce risque est même supérieur 
à la moyenne européenne.

En ce qui concerne les ménages avec enfants, ce sont les familles monoparentales qui sont de loin les plus touchées par la pauvreté, en particulier en Wallonie où près de $40 \%$ de ces ménages sont en situation précaire, contre $20 \%$ en Flandre. Sachant que les familles monoparentales ont dans la plupart de cas à leur tête une femme, ceci expliquerait le taux de pauvreté plus élevé parmi les femmes observé précédemment. Enfin, on remarque aussi un taux de pauvreté plus élevé parmi les familles nombreuses (couples avec 3 enfants ou plus) en Flandre (18,6\%), contrairement à ce qui est constaté pour les autres ménages (autres couples avec enfants).

\section{LE CONFORT DU LOGEMENT COMME INDICATEUR D'INCLUSION SOCIALE}

Dans la section précédente nous avons identifié les différents groupes à risque de pauvreté au sein de la population. Étant donné que l'indicateur utilisé est basé exclusivement sur des informations de type financier recueillies par voie d'enquêtes, il est possible que ces informations soient sujettes à des biais systématiques dus probablement à des erreurs volontaires ou involontaires. Mais plus fondamentalement, il faut reconnaître que le revenu disponible du ménage, même mesuré de manière précise, ne reflète pas le niveau de vie des individus dans son ensemble.

En effet, il se peut qu'indépendamment du niveau de revenu, qui peut d'ailleurs subir de fortes variations dans le temps, le niveau de vie du ménage et des individus qui le composent soit affecté par de privations sur d'autres plans. Notamment par la disponibilité, ou non, de biens ou de services de base qui jouent un rôle déterminant sur la qualité de vie.

Un de ces biens est sans doute la qualité du logement. C'est la raison pour laquelle nous allons analyser dans cette section les informations disponibles, recueillies dans le cadre de l'enquête EU-SILC, portant sur le manque du confort du logement.

Le tableau 3 rapporte le pourcentage de la population vivant dans des logements avec manque de confort, celui-ci étant défini par l'existence d'un des problèmes matériels suivants : un toit non étanche ; l'absence d'un système de chauffage adéquat ; la présence d'humidité et moisissures ; des portes et fenêtres délabrées et/ou un manque d'espace. On retrouve sur ce tableau les mêmes catégories que celles présentées dans le tableau 2, plus une nouvelle catégorie qui fait la distinction selon la nationalité du chef du ménage. Malheureusement, nous ne disposons pas des informations comparables au niveau de l'Europe des 15.

Que constate-t-on?

Tout d'abord, on remarquera l'écart important (du simple au double) en termes de manque de confort du logement entre la Flandre et la Wallonie. Ceci est loin d'être une coïncidence étant donné que cette différence est du même signe et du même ordre que celle observée précédemment au niveau du risque de pauvreté. 
Tableau 3 : Pourcentage de la population vivant dans un logement avec manque de confort en 2005

\begin{tabular}{|c|c|c|c|c|}
\hline Catégorie & Classes & Wallonie & Flandre & Belgique \\
\hline \multirow[t]{2}{*}{ Sexe } & Hommes & 37,1 & 21,5 & 28,7 \\
\hline & Femmes & 39,0 & 22,6 & 30,1 \\
\hline \multirow[t]{5}{*}{ Âge } & $0-17$ & 45,4 & 28,6 & 37,6 \\
\hline & $18-24$ & 38,2 & 22,9 & 30,7 \\
\hline & $25-49$ & 38,7 & 20,6 & 28,8 \\
\hline & $50-64$ & 36,5 & 17,7 & 25,0 \\
\hline & $65+$ & 28,1 & 21,8 & 24,5 \\
\hline \multirow{6}{*}{ Statut d'activité } & Actifs & 32,0 & 17,6 & 23,0 \\
\hline & - travailleur salarié & 32,7 & 17,4 & 23,1 \\
\hline & - indépendant & 27,1 & 19,5 & 22,4 \\
\hline & Chômeurs & 54,6 & 26,9 & 42,9 \\
\hline & Retraités & 30,7 & 21,4 & 24,9 \\
\hline & Autres inactifs & 40,4 & 23,4 & 32,3 \\
\hline \multirow{11}{*}{ Type de ménage } & Sans enfants dépendants & 34,2 & 19,2 & 25,0 \\
\hline & - 1 personne & 41,6 & 27,7 & 33,6 \\
\hline & - 2 personnes <65 ans & 32,4 & 15,4 & 21,6 \\
\hline & - 2 personnes, au moins une $65+$ ans & 22,5 & 20,0 & 21,5 \\
\hline & - autres & 37,7 & 13,4 & 20,5 \\
\hline & Avec enfants dépendants & 41,3 & 24,8 & 33,4 \\
\hline & - ménage monoparental & 63,5 & 38,0 & 50,9 \\
\hline & - couple + 1 enfant & 36,0 & 14,9 & 24,0 \\
\hline & - couple + 2 enfants & 33,2 & 15,0 & 21,6 \\
\hline & - couple +3 enfants ou + & 39,6 & 39,4 & 43,5 \\
\hline & - autres & 46,3 & 30,6 & 40,4 \\
\hline \multirow[t]{2}{*}{ Nationalité } & UE25 & 35,8 & 19,8 & 26,5 \\
\hline & Hors UE25 & 64,8 & 44,5 & 55,1 \\
\hline Total & & 38,1 & 22,0 & 29,4 \\
\hline
\end{tabular}

Source : Groupe de travail Indicateurs PANincl, SPF Sécurité Sociale. À partir des données recueillies dans le cadre de EU-SILC (European Union - Survey on Income and Living Conditions).

Ensuite, on notera aussi que plusieurs des tendances observées au niveau du taux de pauvreté se retrouvent ici. Dans la plupart des cas, ce sont d'ailleurs les mêmes catégories qui se retrouvent opposées : les femmes par rapport aux hommes, les jeunes par rapport aux aînés, les chômeurs et autres inactifs (hors retraités) par rapport aux personnes actives, les familles monoparentales par rapport aux couples avec enfants.

Enfin, on ne peut pas s'empêcher de remarquer qu'en Wallonie, pour certaines catégories de la population, plus de la moitié des personnes déclarent vivre dans un logement inconfortable. C'est le cas des familles monoparentales 
(63,5 \%), mais également des chômeurs (54,6 \%) et des personnes de nationalité non européenne $(64,8)$.

\section{CONCLUSIONS}

La Méthode de Coordination Ouverte (MOC) adoptée par l'Union européenne en l'année 2000 a donné lieu à une collecte d'informations homogènes par voie d'enquête qui ont permis à leur tour le calcul d'indicateurs d'inclusion sociale. Même si les régions européennes n'ont pas été associées en tant que telles à la MOC, la disponibilité de ces informations ouvrent la possibilité pour elles de se situer par rapport aux autres, de fixer des objectifs à atteindre et d'ensuite mesurer l'impact des réformes introduites.

Dans cet article nous avons illustré l'intérêt de cette démarche en prenant comme exemple le cas de la Région wallonne. Nous avons ainsi pu montrer que du point de vue des indicateurs d'inclusion sociale, la Wallonie était située en 2005 en dessous de la moyenne de l'Europe des 15, au même niveau que la Grèce, et que c'était surtout au niveau des taux de pauvreté et de chômage de long terme, deux des indicateurs primaires d'inclusion sociale, qu'elle affichait des résultats défavorables.

L'étape suivante a consisté à identifier les catégories à risque d'exclusion sociale dans la population en nous servant du taux de pauvreté et d'un indicateur du confort du logement. Les résultats montrent une forte coïncidence des deux indicateurs. D'une part, l'écart entre la Flandre et la Wallonie est dans les deux cas substantiel. D'autre part, dans les deux cas, les mêmes catégories ont été identifiées comme groupes à risque : familles monoparentales, chômeurs et autres inactifs hors retraités. Néanmoins, nous avons aussi constaté que de manière générale, la situation des personnes âgées est également devenue préoccupante aujourd'hui, ce qui n'était pas le cas une dizaine d'années auparavant.

Malheureusement, les indicateurs dont nous disposons étant établis pour l'année 2005, ils ne nous permettent pas d'évaluer les actions du gouvernement wallon menées durant la dernière législature. À titre d'exemple, citons la création d'emploi via des entreprises d'insertion pour des personnes faiblement qualifiées ; l'augmentation du nombre d'agences de mises à l'emploi (Région wallonne, 2005) ; l'augmentation du nombre des logements sociaux disponible et la promotion des Agences Immobilières Sociales.

Nous sommes convaincus que la possibilité de disposer désormais d'indicateurs d'inclusion sociale de manière systématique et homogène est un atout important pour l'évaluation des politiques menées mais également pour l'orientation de ces mêmes politiques pour les rendre plus efficaces. De ce point de vue, on doit regretter le fait que l'ensemble de ces indicateurs ne soit pas disponible pour la Région bruxelloise, ceci en raison de la taille trop petite des échantillons lors des enquêtes nationales. Le peu d'informations disponibles indique en effet que c'est au sein de la région capitale que les problèmes d'exclusion sociale sont plus présents qu'ailleurs. 


\section{BIBLIOGRAPHIE}

Atkinson, Tony, Cantillon, Béatrice, Nolan, Brian (2001), Indicators for Social Inclusion in the European Union, Conference on «Indicators for Social Inclusion : Making Common EU Objectives Work - Anvers, 14-15 septembre 2001.

Delhausse, Bernard, Perelman, Sergio, Pestieau, Pierre (1999), "Portrait social de la Wallonie, une mise à jour », CREPP Working Papers, 99(5), p. 1-32.

Delhausse, Bernard, Perelman, Sergio, Pestieau, Pierre (2002), " Un nouveau portrait social de la Wallonie ", Reflets et Perspectives, 2002(3), p. 7-22.

Guio, Anne-Catherine (2003), « La pauvreté en Belgique, en Flandre et en Wallonie. L'éclairage des indicateurs de Laeken sur la pauvreté monétaire ", Tendances économiques, 2003(24), p. 93-107.

Guio, Anne-Catherine (2006), "Pauvreté monétaire en Belgique, en Flandre et en Wallonie ", IWEPS Discussion Papers, 2006(04), p. 1-35.

Lefèbvre, Mathieu, Perelman, Sergio et Pestieau, Pierre (2005), « Faut-il un ou plusieurs indicateurs d'exclusion sociale ", Revue Belge de Sécurité Sociale, 2005(1), p. 59-76.

Région Wallonne (2005), «Plan Stratégique n³ - Inclusion Sociale », http://gov.wallonie. be/IMG/pdf/pst3.pdf.

\section{ANNEXE}

\begin{tabular}{|l|l|}
\hline \multicolumn{2}{|c|}{ Définition des indicateurs d'inclusion sociale } \\
\hline Taux de pauvreté & $\begin{array}{l}\text { Taux de risque de pauvreté après transferts sociaux : Proportion de personnes dont le } \\
\text { revenu disponible équivalent après transferts sociaux se situe en dessous du seuil de } \\
\text { risque de pauvreté fixé à } 60 \% \text { du revenu disponible équivalent médian national (après } \\
\text { transferts sociaux). }\end{array}$ \\
\hline $\begin{array}{l}\text { Inégalité des revenus } \\
\text { (S20/S80) }\end{array}$ & $\begin{array}{l}\text { Inégalité de répartition des revenus, rapport interquintile de revenu : Rapport entre } \\
\text { la part du revenu total (S80) perçu par les } 20 \% \text { de la population ayant le revenu le } \\
\text { plus élevé (quintile supérieur) et la part du revenu total (S20) perçu par les 20\% de la } \\
\text { population ayant le revenu le plus bas (quintile inférieur). }\end{array}$ \\
\hline $\begin{array}{l}\text { Chômage de longue } \\
\text { durée }\end{array}$ & $\begin{array}{l}\text { Taux de chômage de longue durée : Chômeurs de longue durée (12 mois et plus) en } \\
\text { pourcentage de la population active totale. }\end{array}$ \\
\hline Désertion scolaire & $\begin{array}{l}\text { Jeunes ayant quitté prématurément l'école : Pourcentage de la population âgée de } \\
18-24 \text { ans ne suivant ni études, ni formation et dont le niveau d'études ne dépasse } \\
\text { pas l'enseignement secondaire inférieur. }\end{array}$ \\
\hline Espérance de vie & $\begin{array}{l}\text { Espérance de vie à la naissance : Nombre d'années qu'une personne peut espérer } \\
\text { vivre. En commençant à l'âge 0. }\end{array}$ \\
\hline
\end{tabular}


- 